\title{
MARKUSCHEVICH BASES AND DUALITY THEORY
}

\author{
BY \\ WILLIAM B. JOHNSON $\left({ }^{1}\right)$
}

\begin{abstract}
Several duality theorems concerning Schauder bases in locally convex spaces have analogues in the theory of Markuschevich bases. For example, a locally convex space with a Markuschevich basis is semireflexive iff the basis is shrinking and boundedly complete.

The strong existence Theorem III.1 for Markuschevich bases allows us to show that a separable Banach space is isomorphic to a conjugate space iff it admits a boundedly complete Markuschevich basis, and that a separable Banach space has the metric approximation property iff it admits a Markuschevich basis which is a generalized summation basis in the sense of Kadec.
\end{abstract}

I. Introduction. In recent years a number of papers have discussed applications of Schauder bases to the duality theory of locally convex spaces. (For example, see [2], [5], [9], [10], and [11].) However, the lack of a good existence theorem for Schauder bases severely limits the applicability of these results. In this paper we discuss a generalization of Schauder bases (called Markuschevich bases or $M$-bases) for which there are good existence theorems. In fact, Markuschevich [8] showed that every separable Banach space admits a $M$-basis and Theorem III.1 gives a better existence theorem for general linear topological spaces.

In §II we introduce the concepts of shrinking and boundedly complete Markuschevich bases. The main results of this section are that a locally convex space with a $M$-basis is semireflexive iff the $M$-basis is shrinking and boundedly complete (Theorem II.6) and that a Banach space which admits a boundedly complete $M$-basis is canonically isomorphic to the adjoint of the coefficient space of the $M$-basis (Theorem II.5). Of course, these theorems have analogues in Schauder basis theory (see [5], [9], and [1]).

Theorem III. 1 shows that every strongly separable, strongly closed, total subspace of the adjoint of a separable linear topological space $X$ is the coefficient space of some countable $M$-basis for $X$. (This result is perhaps implicit in the results of [4], but we include a proof for completeness.) This theorem has several interesting applications. For example, a separable locally convex space has a strongly separable adjoint iff the space admits a countable shrinking $M$-basis (Corollary III.3). A separable Banach space is isomorphic to a conjugate Banach space iff the space admits a boundedly complete $M$-basis (Theorem III.4).

Received by the editors May 5, 1969 and, in revised form, November 17, 1969.

AMS Subject Classifications. Primary 4601, 4610; Secondary 4160.

Key Words and Phrases. Schauder basis, biorthogonal system, metric approximation property, semireflexivity.

( $\left.{ }^{1}\right)$ The author was partially supported by NASA traineeship NsG(T) 35.3 .

Copyright (C) 1970, American Mathematical Society 
In §IV we discuss generalized summation bases, a type of Markuschevich bases introduced by Kadec [6]. We use Theorem III.1 to show that a separable Banach space admits a generalized summation basis iff the space has the metric approximation property.

We use the notation and terminology of [7]. $X$ always represents a Hausdorff linear topological space and $X^{*}$ represents the set of continuous linear functionals on $X$. We assume that $X^{*}$ is endowed with the topology of uniform convergence on $w\left(X, X^{*}\right)$ bounded subsets of $X$, and call this topology on $X^{*}$ the strong topology. When $X^{*}$ is total over $X$, the natural embedding of $X$ into $X^{* *}$ is denoted by "^`".

If $f$ is a function on a set $Z$ and $Y$ is a subset of $Z,\left.f\right|_{Y}$ denotes the restriction of $f$ to $Y$. The linear span of a subset $Y$ of a linear space is denoted by $\operatorname{sp}(Y)$. The domain, range, and null space of a linear operator $T$ are denoted, respectively, by $D(T), R(T)$, and $\operatorname{ker}(T)$.

II. Applications of $M$-bases to duality theory. Let $(X, T)$ be a linear topological space. Recall that a biorthogonal collection $\left\{x_{i}, f_{i}\right\}_{i \in I}$ in $\left(X, X^{*}\right)$ is a Markuschevich basis (M-basis) for $X$ iff $\left\{x_{i}\right\}_{i \in I}$ is fundamental in $(X, T)$ and $\left\{f_{i}\right\}_{i \in I}$ is total over $X$. The strong closure of $\operatorname{sp}\left(\left\{f_{i}\right\}_{i \in I}\right)$ in $X^{*}$ is called the coefficient space of the $M$-basis $\left\{x_{i}, f_{i}\right\}$.

Definition II.1. Let $\left\{x_{i}, f_{i}\right\}_{i \in I}$ be a $M$-basis for $X$. $\left\{x_{i}, f_{i}\right\}$ is said to be shrinking iff $\left\{f_{i}\right\}_{i \in I}$ is strongly fundamental in $X^{*}$. (Equivalently, $\left\{x_{i}, f_{i}\right\}$ is shrinking iff $\left\{f_{i}, \hat{x}_{i}\right\}$ is a $M$-basis for $X^{*}$ when $X^{*}$ is endowed with the strong topology.)

Definition II.2. Let $\left\{x_{i}, f_{i}\right\}_{i \in I}$ be a $M$-basis for $X .\left\{x_{i}, f_{i}\right\}$ is said to be boundedly complete iff whenever $\left\{Y_{d}\right\}$ is a bounded net in $X$ such that for each $i$ in $I, \lim _{d} f_{i}\left(Y_{d}\right)$ exists, there is $x$ in $X$ such that for each $i \in I, f_{i}(x)=\lim _{d} f_{i}\left(Y_{d}\right)$.

Let $\left\{x_{i}, f_{i}\right\}_{i=1}^{\infty}$ be a Schauder basis for a locally convex space $X$. If $\left\{x_{i}, f_{i}\right\}$ is shrinking as a Schauder basis, then obviously it is shrinking as a $M$-basis. It is also easy to see that if $\left\{x_{i}, f_{i}\right\}$ is boundedly complete as a $M$-basis, then it is boundedly complete as a Schauder basis. The converses of these statements are true for uniformly bounded Schauder bases.

THEOREM II.3. Let $\left\{x_{i}, f_{i}\right\}_{i=1}^{\infty}$ be a uniformly bounded Schauder basis for a locally convex space $X$. (1) If $\left\{x_{i}, f_{i}\right\}$ is boundedly complete as a Schauder basis, then it is boundedly complete as a M-basis. (2) If $\left\{x_{i}, f_{i}\right\}$ is shrinking as a M-basis, then it is shrinking as a Schauder basis.

Proof. Let $\left\{S_{n}\right\}_{n=1}^{\infty}$ be the partial sum operators associated with $\left\{x_{i}, f_{i}\right\}$. (That is, $S_{n}(x)=\sum_{i=1}^{n} f_{i}(x) x_{i}$.) We are assuming that $\left\{S_{n}\right\}_{n=1}^{\infty}$ is uniformly bounded.

To prove (1), we let $\left\{Y_{d}: d \in D\right\}$ be as in Definition II.2. For each $n$, $\left\{S_{n}\left(Y_{d}\right): d \in D\right\}$ is a Cauchy net in the finite dimensional Hausdorff space $\left(R\left(S_{n}\right)\right.$, $\left.w\left(R\left(S_{n}\right),\left\{f_{i}\right\}_{i=1}^{n}\right)\right)$, hence $\lim _{d} S_{n}\left(Y_{d}\right)$ exists. Since $\left\{S_{n}\right\}$ is uniformly bounded and $\left\{Y_{d}\right\}$ is bounded, $\left\{\lim _{d} S_{n}\left(Y_{d}\right)\right\}_{n=1}^{\infty}$ is bounded. Since $\left\{x_{i}, f_{i}\right\}$ is boundedly complete as a Schauder basis, $\left\{\lim _{d} S_{n}\left(Y_{d}\right)\right\}_{n=1}^{\infty}$ must converge to, say, $x$. Clearly $f_{i}(x)$ 
$=\lim _{d} f_{i}\left(Y_{d}\right)$, for all $i=1,2,3, \ldots$, so $\left\{x_{i}, f_{i}\right\}$ is boundedly complete as a $M$-basis.

To prove (2), we note that the uniform boundedness of $\left\{S_{n}\right\}$ implies that $\left\{S_{n}^{*}\right\}_{n=1}^{\infty}$ is equicontinuous on $X^{*}$. Hence $A=\left\{x: \lim _{n} S_{n}^{*}(x)=x\right\}$ is closed in $X^{*}$. Since $A$ obviously contains $\operatorname{sp}\left(\left\{f_{i}\right\}\right)$ and $\left\{f_{i}\right\}$ is fundamental in $X^{*}, A$ must equal $X^{*}$. Then $\left\{f_{i}, \hat{x}_{i}\right\}_{i=1}^{\infty}$ is a Schauder basis for $X^{*}$, which is to say that $\left\{x_{i}, f_{i}\right\}$ is a shrinking Schauder basis for $X$.

The hypothesis in Theorem II. 3 that $\left\{x_{i}, f_{i}\right\}_{i=1}^{\infty}$ is a uniformly bounded Schauder basis is necessary. Indeed, let $Y$ be any separable, infinite dimensional Banach space and let $\left\{x_{i}, f_{i}\right\}_{i=1}^{\infty}$ be any $M$-basis for $Y$ which is not a Schauder basis for $Y$. Let $X=\left\{x \in Y:\left\{\sum_{i=1}^{n} f_{i}(x) x_{i}\right\}_{n=1}^{\infty}\right.$ converges weakly to $\left.x\right\}$. Let $X$ be endowed with the $w\left(X, Y^{*}\right)$ topology. It is easy to see that $\left\{x_{i}, f_{i}\right\}_{i=1}^{\infty}$ is boundedly complete as a Schauder basis for $X .\left\{x_{i}, f_{i}\right\}_{i=1}^{\infty}$ is not boundedly complete as a $M$-basis for $X$ because $X$ is a proper subspace of $Y$. Also, $\left\{x_{i}, f_{i}\right\}_{i=1}^{\infty}$ is obviously a shrinking $M$-basis for $X .\left\{x_{i}, f_{i}\right\}_{i=1}^{\infty}$ is not a shrinking Schauder basis for $X$ because $\left\{f_{i}\right\}_{i=1}^{\infty}$ is not even a basic sequence in $Y^{*}\left(=X^{*}\right)$ and $s\left(Y^{*}, Y\right)=s\left(Y^{*}, X\right)$.

Results from Schauder basis theory suggest that "shrinking" and "boundedly complete" should be dual concepts. Under certain circumstances, this is the case.

THEOREM II.4. Let $\left\{x_{i}, f_{i}\right\}_{i \in I}$ be a shrinking M-basis for a locally convex evaluable space $X$. Then $\left\{f_{i}, \hat{x}_{i}\right\}_{i \in I}$ is a boundedly complete M-basis for $X^{*}$.

Proof. Let $\left\{Y_{d}\right\}$ be a bounded net in $X^{*}$ such that for each $i$ in $I, \lim _{d} \hat{x}_{i}\left(Y_{d}\right)$ exists. Note that $\left\{Y_{d}\right\}$ is equicontinuous on $X$ because $X$ is evaluable, hence $\left\{Y_{d}\right\}$ has a weak* cluster point, say, $y$. Clearly $\hat{x}_{i}(y)=\lim _{d} \hat{x}_{i}\left(Y_{d}\right)$, for all $i$ in $I$, so $\left\{f_{i}, \hat{x}_{i}\right\}$ is boundedly complete.

THEOREM II.5. Let $\left\{x_{i}, f_{i}\right\}_{i \in I}$ be a boundedly complete M-basis for a Banach space $X$. Let $Y$ be the coefficient space of the basis $\left\{x_{i}, f_{i}\right\}$. Then the canonical embedding of $X$ into $Y^{*}$ is an isomorphism of $X$ onto $Y^{*}$. Hence $\left\{f_{i},\left.\hat{x}_{i}\right|_{Y}\right\}_{i \in I}$ is a shrinking $M$-basis for $Y$.

Proof. Note that the canonical embedding of $X$ into $Y^{*}$ is one-to-one, because $Y$ is total over $X$. It is norm decreasing, hence continuous. We show that it is onto $Y^{*}$ (and hence an isomorphism by the open mapping theorem).

Let $G$ be in $Y^{*}$. Let $D$ be the collection of finite subsets of $I$, and direct $D$ by inclusion. By Helley's theorem (cf., e.g., [12, p. 103]), for each $d$ in $D$ there is $Y_{d}$ in $X$ such that for each $i$ in $d, f_{i}\left(Y_{d}\right)=G\left(f_{i}\right)$, and $\left\|Y_{d}\right\| \leqq\|G\|+1$. Since $\left\{x_{i}, f_{i}\right\}$ is boundedly complete, there is $x$ in $X$ such that for each $i$ in $I, \lim _{d} f_{i}\left(Y_{d}\right)=f_{i}(x)$. Clearly $f_{i}(x)=G\left(f_{i}\right)$, for all $i$ in $I$. Since $\left\{f_{i}\right\}$ is fundamental in $Y$ and both $\hat{x}$ and $G$ are continuous on $Y, f(x)=G(f)$, for all $f$ in $Y$. Thus the canonical embedding of $X$ into $Y^{*}$ is onto.

Since $\left\{x_{i}\right\}$ is fundamental in $X,\left\{\left.\hat{x}_{i}\right|_{Y}\right\}$ is fundamental in $Y^{*}$, and thus $\left\{f_{i},\left.\hat{x}_{i}\right|_{Y}\right\}$ is shrinking. This completes the proof.

Historically, a major reason for considering shrinking and boundedly complete 
Schauder bases was to characterize reflexivity (see [5] and [9]). Similarly, semireflexivity is characterized by the existence of a boundedly complete, shrinking $M$-basis.

THEOREM II.6. Let $\left\{x_{i}, f_{i}\right\}_{i \in I}$ be a M-basis for a locally convex space $X . X$ is semireflexive iff $\left\{x_{i}, f_{i}\right\}$ is both shrinking and boundedly complete.

Proof. Suppose first that $X$ is semireflexive. $\left\{f_{i}\right\}$ is total over $X$, hence is weak* fundamental in $X^{*}$, hence is weakly fundamental in $X^{*}$, hence is fundamental in $X^{*}$. Thus $\left\{x_{i}, f_{i}\right\}$ is shrinking. Now let $\left\{Y_{d}\right\}$ be a bounded net as in Definition II.2. Since $X$ is semireflexive, $\left\{Y_{d}\right\}$ has a weak cluster point, say, $x$. Clearly $f_{i}(x)$ $=\lim _{d} f_{i}\left(Y_{d}\right)$, for all $i$ in $I$, so that $\left\{x_{i}, f_{i}\right\}$ is boundedly complete.

To go the other way, suppose that $\left\{x_{i}, f_{i}\right\}$ is shrinking and boundedly complete. To show that $X$ is semireflexive, it is sufficient to show that bounded, weakly Cauchy nets in $X$ are weakly convergent. Let $\left\{Y_{d}\right\}$ be a bounded, weakly Cauchy net in $X$. Clearly $\lim _{d} f_{i}\left(Y_{d}\right)$ exists for each $i$ in $I$, hence by the boundedly complete assumption there is $x$ in $X$ such that for all $i$ in $I, f_{i}(x)=\lim _{d} f_{i}\left(Y_{d}\right)$. We need to show that $\left\{Y_{d}\right\}$ weakly converges to $x$. Now $\left\{Y_{d}\right\}$ is bounded, so $\left\{\hat{Y}_{d}\right\}$ is equicontinuous on $X^{*}$. $\left\{\hat{Y}_{d}\right\}$ converges to $\hat{x}$ pointwise on the subset $\left\{f_{i}\right\}$ of $X^{*}$. By the shrinking assumption, $\left\{f_{i}\right\}$ is fundamental in $X^{*}$, hence $\left\{\hat{Y}_{d}\right\}$ converges to $\hat{x}$ pointwise on $X^{*}$. That is, $\left\{Y_{d}\right\}$ converges weakly to $x$. This completes the proof.

III. Existence theorem for countable Markuschevich bases. Unfortunately, there is no general existence theorem for Schauder bases. In contrast to this situation, Theorem III.1 provides a very fine existence theorem for $M$-bases.

THEOREM III.1. Let $X$ be separable and let $Y$ be a closed, separable, total subspace of $X^{*}$. Then $X$ admits a M-basis $\left\{y_{i}, g_{i}\right\}_{i=1}^{\infty}$ whose coefficient space is $Y$.

Proof. Let $\left\{x_{i}\right\}_{i=1}^{\infty}$ be a fundamental subset of $X$ and let $\left\{f_{i}\right\}_{i=1}^{\infty}$ be a fundamental subset of $Y$. Note that $\left\{f_{i}\right\}_{i=1}^{\infty}$ is total over $X$. Hence we can assume, without loss of generality, that $f_{1}\left(x_{1}\right) \neq 0$.

Let $y_{1}=x_{1}, g_{1}=f_{1} / f_{1}\left(x_{1}\right), k(1)=1$.

Suppose that $\left\{y_{i}, g_{i}\right\}_{i=1}^{k(n)}$ have been defined so that

(1) $\left\{y_{i}, g_{i}\right\}_{i=1}^{k(n)}$ is biorthogonal;

(2) $\operatorname{sp}\left(\left\{y_{i}\right\}_{i=1}^{k(n)}\right) \supset \operatorname{sp}\left(\left\{x_{i}\right\}_{i=1}^{n}\right)$;

(3) $Y \supset \operatorname{sp}\left(\left\{g_{i}\right\}_{i=1}^{k(n)}\right) \supset \operatorname{sp}\left(\left\{f_{i}\right\}_{i=1}^{n}\right)$.

A. If $x_{n+1}$ is in $\operatorname{sp}\left(\left\{y_{i}\right\}_{i=1}^{k(n)}\right)$, we let $k=k(n)$ and proceed to B. If $x_{n+1}$ is not in $\operatorname{sp}\left(\left\{y_{i}\right\}_{i=1}^{k(n)}\right)$, we let $k=k(n)+1$, and let $y_{k}=x_{n+1}-\sum_{i=1}^{k(n)} g_{i}\left(x_{n+1}\right) y_{i}$. (1) implies that $g_{i}\left(y_{k}\right)=0$, for each $i \leqq k(n)$. By using the Hahn-Banach theorem in the space $(X, w(X, Y))$, we can find $g_{k}$ in $Y$ such that $g_{k}\left(y_{k}\right)=1$ and $g_{k}\left(y_{i}\right)=0$ for each $i \leqq k(n)$. Note that $\left\{y_{i}, g_{i}\right\}_{i=1}^{k}$ is biorthogonal, $\operatorname{sp}\left(\left\{y_{i}\right\}_{i=1}^{k}\right) \supset \operatorname{sp}\left(\left\{x_{i}\right\}_{i=1}^{n+1}\right)$, and $Y \supset \operatorname{sp}\left(\left\{g_{i}\right\}_{i=1}^{k}\right)$. Now proceed to B.

B. If $f_{n+1}$ is in $\operatorname{sp}\left(\left\{g_{i}\right\}_{i=1}^{k}\right)$, let $k(n+1)=k$. If $f_{n+1}$ is not in $\operatorname{sp}\left(\left\{g_{i}\right\}_{i=1}^{k}\right)$, let $k(n+1)$ 
$=k+1$, and let $g_{k(n+1)}=f_{n+1}-\sum_{i=1}^{k} f_{n+1}\left(y_{i}\right) g_{i}$. By using the Hahn-Banach theorem in the space $(Y, w(Y, X))$, we can find $y_{k(n+1)}$ in $X$ such that $g_{k(n+1)}\left(y_{k(n+1)}\right)=1$ and $g_{i}\left(y_{k(n+1)}\right)=0$ for all $i \leqq k$.

We have thus extended the sequence $\left\{y_{i}, g_{i}\right\}_{i=1}^{k(n)}$ to a sequence $\left\{y_{i}, g_{i}\right\}_{i=1}^{k(n+1)}$ such that $\left\{y_{i}, g_{i}\right\}_{i=1}^{k(n+1)}$ satisfies (1), (2), and (3) if $n+1$ is substituted for $n$. The sequence $\left\{y_{i}, g_{i}\right\}_{i=1}^{\infty}$ obviously satisfies the conclusion of the theorem.

REMARK III.2. Theorem III.1 shows that every separable linear topological space which admits a countable total family of continuous linear functionals must also admit a countable $M$-basis. Unfortunately, there are separable locally convex spaces which do not admit a countable total family of continuous linear functionals. (An example is the product of $\aleph_{1}$ copies of the real line with the product topology.) Of course, such spaces do not admit countable $M$-bases.

One instance of Theorem III.1 is of particular interest:

Corollary III.3. Suppose that $X$ is separable and $X^{*}$ is total over $X$. Then $X$ admits a countable shrinking $M$-basis iff $X^{*}$ is strongly separable.

THEOREM III.4. Let $X$ be a separable Banach space. $X$ is isomorphic to a conjugate Banach space iff $X$ admits a boundedly complete M-basis.

Proof. If $X$ is isomorphic to $Y^{*}$, then $Y$ is separable, so that the conclusion follows from Corollary III. 3 and Theorem II.4. Conversely, if $X$ admits a boundedly complete $M$-basis, the conclusion follows from Theorem II.5.

Let $X$ be a Banach space. Recall that a subspace $Y$ of $X^{*}$ is norming iff there is $k>0$ such that for all $x$ in $X,\|x\| \leqq k$ sup $\{|f(x)|: f \in Y,\|f\| \leqq 1\}$. Equivalently, $Y$ is norming iff the canonical mapping of $X$ into $Y^{*}$ is an isomorphism of $X$ into $Y^{*}$. Note that Theorem II.5 shows that the coefficient space of a boundedly complete $M$-basis is norming. However, there are $M$-bases whose coefficient spaces are not norming. This follows from Theorem III.1 and the well-known fact that there are closed total subspaces of $c_{0}^{*}$ which are not norming. On the other hand, if $X$ is separable it is easy to see that $X^{*}$ contains a separable norming subspace. It thus follows from Theorem III.1 that every separable Banach space admits a $M$-basis whose coefficient space is norming.

For many applications, it is desirable to have a stronger existence theorem for $M$-bases than Theorem III.1. In particular, which nonseparable Banach spaces admit $M$-bases? (Dyer [3] has noted that for $T$ uncountable, $m(T)$ has no $M$-bases.) Let $X$ be a separable Banach space. Is there a $M$-basis $\left\{x_{i}, f_{i}\right\}$ for $X$ such that $\left\{x_{i}\right\}$ is bounded in $X,\left\{f_{i}\right\}$ is bounded in $X^{*}$, and the coefficient space of $\left\{x_{i}, f_{i}\right\}$ is norming?

IV. Generalized summation bases in spaces with the metric approximation property. In this section we assume that $X$ is a separable Banach space, $\left\{x_{i}, f_{i}\right\}_{i=1}^{\infty}$ is a $M$-basis for $X$, and $\left\{S_{n}\right\}_{n=1}^{\infty}$ is the sequence of operators defined by $S_{n}(x)=\sum_{i=1}^{n} f_{i}(x) x_{i}$. $I$ is the identity operator on $X$. 
Following Kadec [6] we say that $\left\{x_{i}, f_{i}\right\}$ is a generalized summation basis (g.s.b.) for $X$ iff there is a sequence $\left\{T_{n}\right\}_{n=1}^{\infty}$ of linear operators with $R\left(T_{n}\right) \subset D\left(T_{n}\right)=$ sp $\left(\left\{x_{i}\right\}_{i=1}^{n}\right)$ such that the sequence $\left\{T_{n} S_{n}\right\}_{n=1}^{\infty}$ of linear operators on $X$ converges pointwise to $I$. Kadec pointed out that not every countable $M$-basis is a g.s.b. Indeed, this follows from the comments at the end of \$III and the easily verified fact that the coefficient space of a g.s.b. is norming (see [6] and [4]).

It is not known whether every separable Banach space admits a g.s.b. Note that the existence of a g.s.b. for $X$ implies that $X$ has the metric approximation property-i.e., that there is a sequence of continuous linear operators of finite range (but not necessarily of norm 1) on $X$ which converges pointwise to $I$. In fact, Theorem IV.1 shows that the metric approximation property is equivalent to the existence of a g.s.b.

THEOREM IV.1. Let $X$ be a separable Banach space which has the metric approximation property. Then $X$ admits a generalized summation basis.

Proof. Let $\left\{L_{n}\right\}_{n=1}^{\infty}$ be a sequence of linear operators of finite range on $X$ which converges pointwise to $I$. Let $\left\{x_{i}, f_{i}\right\}_{i=1}^{\infty}$ be any $M$-basis for $X$ such that the coefficient space of $\left\{x_{i}, f_{i}\right\}$ contains $\bigcup_{n=1}^{\infty} R\left(L_{n}^{*}\right)$. (By Theorem III.1, such a $M$-basis exists.)

Write $L_{1}(x)=\sum_{i=1}^{p} g_{i}(x) y_{i}$, where $\left\{y_{i}\right\}_{i=1}^{p} \subset R\left(L_{1}\right)$ and $\left\{g_{i}\right\}_{i=1}^{p} \subset R\left(L_{1}^{*}\right)$. If $\varepsilon>0$, there is a positive integer $n(1)$ such that for each $i \leqq p$, there are $\bar{x}_{i}$ in $\operatorname{sp}\left(\left\{x_{i}\right\}_{i=1}^{n(1)}\right)$ and $\bar{f}_{i}$ in $\operatorname{sp}\left(\left\{f_{i}\right\}_{i=1}^{n(1)}\right)$ such that $\left\|\bar{x}_{i}-y_{i}\right\|<\varepsilon$ and $\left\|\bar{f}_{i}-g_{i}\right\|<\varepsilon$.

Let $T_{n(1)}(x)=\sum_{i=1}^{p} \bar{f}_{i}(x) \bar{x}_{i}$. Note that

$$
\begin{aligned}
\left\|T_{n(1)}(x)-L_{1}(x)\right\| & =\left\|\sum_{i=1}^{p}\left(\bar{f}_{i}(x)-g_{i}(x)\right) \bar{x}_{i}+\sum_{i=1}^{p} g_{i}(x)\left(\bar{x}_{i}-y_{i}\right)\right\| \\
& \leqq\left[\sum_{i=1}^{p}\left\|\bar{f}_{i}-g_{i}\right\|\left\|\bar{x}_{i}\right\|+\left\|g_{i}\right\|\left\|\bar{x}_{i}-y_{i}\right\|\right]\|x\| .
\end{aligned}
$$

Thus if $\varepsilon$ is sufficiently small, we can assure that $\left\|T_{n(1)}-L_{1}\right\|<1$.

Note that $T_{n(1)}=T_{n(1)} S_{n(1)}$ and $R\left(T_{n(1)}\right) \subset \operatorname{sp}\left(\left\{x_{i}\right\}_{i=1}^{n(1)}\right)$.

A simple extension of this argument shows that there is an increasing sequence $\{n(i)\}_{i=1}^{\infty}$ of positive integers and a sequence $\left\{T_{n(i)}\right\}_{i=1}^{\infty}$ of linear operators on $X$ such that for each $i$,

$$
\left\|T_{n(i)}-L_{i}\right\|<1 / i, \quad R\left(T_{n(i)}\right) \subset \operatorname{sp}\left(\left\{x_{j}\right\}_{j=1}^{n(i)}\right), \quad \text { and } \quad T_{n(i)}=T_{n(i)} S_{n(i)} .
$$

$\left\{T_{n(i)}\right\}_{i=1}^{\infty}$ converges pointwise to $I$ because $\left\{\left\|T_{n(i)}-L_{i}\right\|\right\}_{i=1}^{\infty}$ converges to 0 and $\left\{L_{i}\right\}_{i=1}^{\infty}$ converges pointwise to $I$.

We complete the sequence $\left\{T_{j}\right\}_{j=1}^{\infty}$ by defining

$$
\begin{aligned}
T_{j} & =S_{j}, & & \text { if } j<n(1), \\
& =T_{n(i)}, & & \text { if } n(i) \leqq j<n(i+1) .
\end{aligned}
$$

Note that for each $j, T_{j}=T_{j} S_{j}$. We have seen that $\left\{T_{j}\right\}_{j=1}^{\infty}$ converges pointwise to $I$, 
so $\left\{T_{j} S_{j}\right\}_{j=1}^{\infty}$ converges pointwise to $I$. Clearly $R\left(T_{j}\right) \subset \operatorname{sp}\left(\left\{x_{i}\right\}_{i=1}^{j}\right)$, so we have shown that $\left\{x_{i}, f_{i}\right\}_{i=1}^{\infty}$ is a g.s.b. for $X$.

Theorem II.6 and the proof of Theorem IV.1 yield a (slight) strengthening of Theorem 4 of [6].

COROLlaRY IV.2. Let $X$ be a separable reflexive Banach space which has the metric approximation property. Then every $M$-basis for $X$ is a g.s.b.

\section{REFERENCES}

1. Leon Alaoglu, Weak topologies of normed linear spaces, Ann. of Math. (2) 41 (1941), 252-267. MR 1, 241.

2. E. Dubinsky and J. R. Retherford, Schauder bases and Köthe sequence spaces, Trans. Amer. Math. Soc. 130 (1968), 265-280. MR 38 \#510.

3. J. A. Dyer, Generalized Markuschevich bases, Israel J. Math. 7 (1969), 51-59.

4. V. F. Gapoškin and M. I. Kadec, Operator bases in Banach spaces, Mat. Sb. 61 (103) (1963), 3-12. (Russian) MR 27 \#1810.

5. R. C. James, Bases and reflexivity of Banach spaces, Ann. of Math. (2) 52 (1950), 518-527. MR 12, 616.

6. M. I. Kadec, Bi-orthogonal systems and summation bases, Funkcional. Anal. i Primenen. (Trudy 5 Konf. po Funkcional'nomu Analizu i ego Primeneniju) Izdat. Akad. Nauk Azerbaĭdžan. SSR, Baku 1961, pp. 106-108. (Russian) MR 26 \#2858.

7. J. L. Kelley, I. Namioka, et al., Linear topological spaces, The University Series in Higher Math., Van Nostrand, Princeton, N. J., 1963. MR 29 \#3851.

8. A. Markuschevich, Sur les bases (au sens large) dans les espaces linéaires, Dokl. Akad. Nauk SSSR 41 (1943), 227-229. MR 6, 69.

9. J. R. Retherford, Bases, basic sequences and reflexivity of linear topological spaces, Math. Ann. 164 (1966), 280-285. MR 33 \#6351.

10. J. R. Retherford and E. Dubinsky, Schauder bases in compatible topologies, Studia Math. 28 (1966/67), 221-226. MR 36 \#640.

11. Ivan Singer, Basic sequences and reflexivity of Banach spaces, Studia Math. 21 (1961/62), 351-369. MR 26 \#4155.

12. Albert Wilansky, Functional analysis, Blaisdell, Waltham, Mass., 1963. MR 30 \#425.

Iowa State University, AMEs, IOWA 50010

UNIVERSITY OF HOUSTON, Houston, TEXAS 77004 